\title{
Recursive Least Squares Run-to-Run Control with Time-Varying Metrology Delays
}

\author{
Shu-Kai Fan ${ }^{\dagger}$ \\ Department of Industrial Engineering and Management, \\ Yuan Ze University, Tao Yuan County, Taiwan 320, (ROC) \\ E-mail: simonfan@saturn.yzu.edu.tw \\ Yuan-Jung Chang \\ Department of Industrial Engineering and Management, \\ Yuan Ze University, Tao Yuan County, Taiwan 320, (ROC) \\ E-mail: s968908@mail.yzu.edu.tw
}

Received, March 10, 2010; Revised, July 2, 2010; Accepted, July 14, 2010

\begin{abstract}
This article investigates how to adaptively predict the time-varying metrology delay that could realistically occur in the semiconductor manufacturing practice. Metrology delays pose a great challenge for the existing run-to-run (R2R) controllers, driving the process output significantly away from target if not adequately predicted. First, the expected asymptotic double exponentially weighted moving average (DEWMA) control output, by using the EWMA and recursive least squares (RLS) prediction methods, is derived. It has been found that the relationships between the expected control output and target in both estimation methods are parallel, and six cases are addressed. Within the context of time-varying metrology delay, this paper presents a modified recursive least squares-linear trend (RLS-LT) controller, in combination with runs test. Simulated single inputsingle output (SISO) R2R processes subject to various time-varying metrology delay scenarios are used as a testbed to evaluate the proposed algorithms. The simulation results indicate that the modified RLS-LT controller can yield the process output more accurately on target with smaller mean squared error (MSE) than the original RLSLT controller that only deals with constant metrology delays.
\end{abstract}

Keywords: Run-to-Run(R2R) Control, Time-Varying Metrology Delay, Recursive Least Squares(RLS), Exponentially Weighted Moving Average(EWMA) Control

\section{INTRODUCTION AND BACKGROUND}

Run-to-Run (R2R) control has been successfully applied to a number of semiconductor applications, including chemical mechanical planarization (CMP), chemical deposition, plasma etching, and photolithography, among others. Moyne et al. (2001) defined R2R control as a form of discrete process and machine control where the product recipe with respect to a particular process is modified ex situ, that is, between machine runs, so as to compensate for process drift, shift, and disturbances. In a word, the control of this kind is deemed a supervisory controller regulating the recipe (or called set-point) of the automatic controller from run to run that attempts to maintain the process output on target and reduce the variability simultaneously. It is noted in del Castillo and Hurwitz (1997) that R2R control basically consists of two major components. First, a regression model is con- structed by means of offline experiments using designed experiments, response surface methods, or historical data. Most of the R2R controllers are built on a linear model, but in some circumstances, nonlinearities have to be taken into account (del Castillo and Yeh, 1998). Second, the regression model developed offline is updated based on most recently observed process data and then used to update the recipe for the next run. Exponentially weighted moving average (EWMA), double EWMA (DEWMA), and recursive least squares (RLS) filters have been widely recognized methods for online estimation in the R2R practice. The focus of this paper will be placed on the process control based on the assumption that the regression model is found statistically fit to the process data.

Most of processes in the semiconductor manufacturing contain quality-related parameters that cannot be measured in situ. Post measurements are performed only

$\dagger$ : Corresponding Author 
after a run is completed, and the recipe is updated before the next run is processed (Good and Qin, 2006). Due to the production demand in throughput and constraint in metrology capacity, it is hardly feasible to provide realtime measurement data to the controller as the recipe is updated for executing the next run. Metrology delay is a natural problem for the implementation of advanced process control in semiconductor manufacturing. Wu et al. (2006) proposed several potential solutions to this problem as follows: (i) investment in advanced metrology; (ii) implementation of virtual metrology (VM); (iii) retuning the controller parameters if the metrology delay is changed; (iv) finding the effective time delay strategy in case of time-varying delays. A major motivation for this paper is due to solutions (iii) and (iv). Furthermore, time-varying delays between zero and five would make the control task much more difficult (Wang et al., 2005). As such, this paper wishes to propose a modification to the current R2R controller with metrology delay that could successfully cope with time-varying delays.

The rest of this paper is outlined as follows. Section 2 gives a literature review of the DEWMA and RLS prediction methods, along with their applicability in the SISO R2R system with a fixed metrology delay. Within the realms of the recursive least squares-linear trend (RLS-LT) controller, a particular attention will be placed on the time-varying metrology delay rather than the fixed one that has already been addressed in the literature. Section 3 derives the mathematical properties of the DEWMA and RLS prediction methods. A time update scheme is added to the RLS-LT controller, in combination with runs tests to deal with time-varying metrology delay. Section 4 presents the proposed RLS-LT controller using runs test. Section 5 shows the performance of the proposed method by means of simulations. The summary of the research findings and directions for future research are given in Section 6.

\section{DEWMA AND RLS-LT CONTROLLER WITHOUT METROLOGY DELAYS}

\subsection{Double Exponentially Weighted Moving Aver- age (DEWMA) Control}

Following Ingolfsson and Sachs' (1993) simple EWMA work, Bulter and Stefani (1994) developed a new R2R supervisory controller, termed Predictor Corrector Control (PCC), using a "double" EWMA to alleviate the impact of machine aging and process drift. In the SISO case, the process model assumed by Butler and Stefani in their PCC scheme is

$$
y_{t}=\alpha+\beta u_{t-1}+\delta t+\varepsilon_{t}
$$

where $y_{t}$ is the measured quality characteristic of run $t, u_{t-1}$ is the recipe set at the end of run $(t-1), \delta$ is the average drift per run, and $\left\{\varepsilon_{t}\right\}_{t=1}^{\infty}$ is a white noise sequence. Model (1) assumes a deterministic drift disturbance, given by the term $\delta t+\varepsilon_{t}$. The parameter $\alpha$ models any offset or bias from target and $\beta$ is the input-output gain parameter. If an offline estimate $b$ exists for the gain $\beta$ and $\tau$ is the desired target to achieve, thus the control action can be obtained by:

$$
u_{t}=\frac{\tau-a_{t}-D_{t}}{b},
$$

where $a_{t}=\omega_{1}\left(y_{t}-b u_{t-1}\right)+\left(1-\omega_{1}\right) a_{t-1}$,

with $\omega_{1} \in[0,1]$ and

$$
D_{t}=\omega_{2}\left(y_{t}-b u_{t-1}-a_{t-1}\right)+\left(1-\omega_{2}\right) D_{t-1},
$$

with $\omega_{2} \in[0,1]$.

The double EWMA formulae shown above consist of two geometrically smoothing recursions, the one in (3) used to compensate for the process shift (i.e., step change in the mean; for details, see Ingolfsson and Sachs (1993), Figure 1 Figure 7, for illustration) and the one in (4) used to compensate for the forecast error incurred by a DT disturbance. As discussed in del Castillo (1999), the quality $\left(a_{t}+D_{t}\right)$ provides an asymptotically unbiased one-step-ahead estimate of where the quality characteristic would have drifted in the absence of any control action. In the case of PCC, there are two weights, $\omega_{1}$ and $\omega_{2}$, that need to be defined beforehand. For a considerably large family of drift disturbances, the stability conditions for this SISO PCC feedback controller are shown in del Castillo (1999, 2001). The PCC scheme is not a member of the proportional-integral derivative (PID) family, but contains an integral action that adds robustness and compensates for shifts. This scheme is close, although not equal, to a minimumvariance controller for an ARIMA $(0,2,2)$ process, a process that drifts but not in a monotonic manner (del Castillo, 2001). Typically, the formulae in (3-4) are referred to as the PCC controller.

Later, the DEWMA controller has been modified by Chen and Guo (2001). The difference is merely on the estimate of intercept term, $a_{t}$. Their controller estimates the intercept using the following formula:

$$
a_{t}=\omega_{1}\left(y_{t}-b u_{t-1}\right)+\left(1-\omega_{1}\right)\left(a_{t-1}+D_{t-1}\right)
$$

with $\omega_{1} \in[0,1]$, by which and (4) the process recipe is also defined by (2). Such a control scheme is somewhat distinct from the original PCC formula. That is, in (5), $D_{t-1}$ is added into the first recursion. The asymptotic estimates have more straightforward meanings, as shown below:

$$
\begin{gathered}
a_{t}=\omega_{1} e_{t}+D_{t-1}+a_{t-1}=\omega_{1} \sum_{j=1}^{t} e_{j}+\sum_{j=1}^{t-1} D_{j}+a_{0}+D_{0}, \\
D_{t}=\omega_{2} e_{t}+D_{t-1}=\omega_{2} \sum_{j=1}^{t} e_{j}+D_{0}
\end{gathered}
$$


where $e_{t}=Y_{t}-\tau$. The estimate for $\alpha+\delta(t+1)$ is $a_{t}+$ $D_{t}$, which turns out to be

$$
a_{t}+D_{t}=\omega_{1} \sum_{j=1}^{t} e_{j}+\omega_{2} \sum_{j=1}^{t} \sum_{k=1}^{j} e_{k}+(t+1) D_{0}+a_{0} .
$$

Therefore, it can be shown that $\lim _{t \rightarrow \infty} E\left(D_{t-1}\right)=\delta$ and $E\left(a_{t}\right)=\alpha+\delta t+o(t)$ where $\lim _{t \rightarrow \infty} o(t)=0$. This result is more straightforward than the original PCC formula. Moreover, the two estimates $a_{t}$ and $D_{t}$ used here have clearly defined meanings; that is, $a_{t}$ represents an estimate for $\left(\alpha+\delta_{t}\right) ; D_{t}$ is an estimate for the magnitude of the process drift from the t-th run to the $(\mathrm{t}+1)$-th run. Together, $a_{t}+D_{t}$ is the estimate for $a_{t}+$ $D_{t} \alpha+\delta(t+1)$. Substituting $\lim _{t \rightarrow \infty} o(t)=0$ and $\lim _{t \rightarrow \infty}$ $E\left(D_{t-1}\right)=\delta$ into Equations (1-2), it can be proved that the process output also converges to the desired target $\tau$ (for details, see Chen and Guo, 2001). To avoid ambiguity, the DEWMA controller is henceforth referred to Chen and Guo's version.

\subsection{Recursive Least Squares (RLS) Control}

Wang et al. (2003) and Wang et al. (2005) showed that EWMA and DEWMA were the least-squares estimates for the locally constant mean (CM) model and the locally linear trend (LT) model, respectively, by setting up a specialized forgetting factor scheme. In fact, the locally CM model is identical to (1) without considering $\delta t$; the locally LT model is identical to (1). Only the "locally LT" model is considered in this paper, so the RLS-LT process model (i.e., model (1)) is further expressed by

$$
x_{t}=y_{t}-\beta u_{t-1}=\alpha \cdot 1+\delta t+\varepsilon_{t}=\theta_{1} \cdot 1+\theta_{2} \cdot t+\varepsilon_{t}=\boldsymbol{\varphi}_{t}^{\mathrm{T}} \boldsymbol{\theta}+\varepsilon_{t}
$$

where the off-line estimate $\hat{\beta}$ is kept unchanged throughout, $\boldsymbol{\theta}=\left[\begin{array}{ll}\theta_{1} & \theta_{2}\end{array}\right]^{\mathrm{T}}=\left[\begin{array}{ll}\alpha & \delta\end{array}\right]^{\mathrm{T}}$, and $\boldsymbol{\varphi}_{t}=\left[\begin{array}{ll}1 & t\end{array}\right]^{\mathrm{T}}, \forall t$. The corresponding recursive algorithms are

$$
\begin{aligned}
& \boldsymbol{K}_{t}=\frac{\boldsymbol{P}_{t-1} \boldsymbol{\varphi}_{t}}{\lambda+\boldsymbol{\varphi}_{t}^{\mathrm{T}} \boldsymbol{P}_{t-1} \boldsymbol{\varphi}_{t}}, \\
& \boldsymbol{P}_{t}=\frac{\left(\mathbf{I}-\boldsymbol{K}_{t} \boldsymbol{\varphi}_{t}^{\mathrm{T}}\right) \boldsymbol{P}_{t-1}}{\lambda}, \\
& \hat{\boldsymbol{\theta}}_{t}=\hat{\boldsymbol{\theta}}_{t-1}+\boldsymbol{K}_{t}\left(x_{t}-\boldsymbol{\varphi}_{t}^{\mathrm{T}} \hat{\boldsymbol{\theta}}_{t-1}\right),
\end{aligned}
$$

where $\boldsymbol{\theta}$ is the vector of unknown parameters to be determined; $\boldsymbol{\varphi}$ is the constant vector of regressors; $\boldsymbol{K}$ is the Kalman weighting vector; $\boldsymbol{P}$ is the precision matrix proportional to the variance-covariance matrix of parameter estimates; $\lambda \in[0,1]$ is the RLS-LT discounting factor; I is the identity matrix; $\hat{\boldsymbol{\theta}}$ is the vector of parameter estimates. Thus, the control action of RLS-LT is defined by

$$
u_{t}=\frac{\tau-\hat{\boldsymbol{\theta}}_{\mathrm{t}_{t}}-(t+1) \hat{\boldsymbol{\theta}}_{2_{t}}}{b}
$$

where $\hat{\theta}_{1}$ is the least-squares estimate of $\alpha ; \hat{\theta}_{2}$ is the least-squares estimate of $\delta ; b=\hat{\beta}$ is the estimate of $\beta$. Wang et al. (2005) also proved that both EWMAand RLS-type estimates were asymptotically identical under a particular condition. That is, the transformed recursive algorithms are exactly the DEWMA form with $\omega_{1}=1-\lambda^{2}, \omega_{2}=(1-\lambda)^{2}$ and $x_{t}=y_{t}-\beta u_{t-1}$. The DEW MA cannot be deduced from RLS-LT reversely. The DEWMA control in (4-5) is of a more general form than RLS-LT in (10-13) due to 2 different weighting factors being used. However, this makes the tuning process more difficult. Notice that $\omega_{1}$ and $\omega_{2}$ in (4-5) cannot be chosen arbitrarily since some combinations of $\omega_{1}$ and $\omega_{2}$ can make the system unstable. Compared to DEW MA, the RLS-LT control owns the merit of being easy to implement in practice since only a "single" forgetting factor needs to be tuned. Furthermore, although EWMA -type and RLS-type estimates were proved to have the same asymptotic property as tuning was set to mild changes in the process model, RLS-type estimates responded to process changes faster than EWMA-type estimates (Wang et al., 2005). Therefore, if the discounting factor $(\lambda)$ can be appropriately chosen, then the controlled process output also converges to the desired target $\tau$.

\section{RELATIONSHIP BETWEEN THE EX- PECTED PROCESS OUTPUT AND TARGET IN THE PRESENCE OF ME- TROLOGY DELAYS AND DETERMI- NISTIC DRIFTS}

\subsection{Simulation Study of DEWMA and RLS-LT with Metrology Delays}

The process output is known to be deviated from target if there is metrology delay in the control system (Carlson and DiBiase, 2002 and Chamness et al., 2001). In this section, to gain knowledge of how metrology delays affect the DEWMA and RLS-LT controllers, Monte Carlo simulations are conducted. Suppose that the target value is $\tau=0$, the process offset $\alpha=0$, initial process offset estimate $\hat{\alpha}_{0}=0$, initial process drift estimate $\hat{\delta}_{0}=0$, the model mismatch parameter $\xi=$ $\beta / b=1$ and $\varepsilon_{t} \sim N\left(0,1^{2}\right)$. Wang et al. (2005) reported that as $\lambda>0.6$ was chosen, the RLS-LT was not sensitive to model mismatch as compared to EWMA and DEWMA. Here, the discounting factor of RLS-LT controller in (11) is set to $\lambda=0.85$. Based on a fair comparison basis that only a single tuning factor is considered, the weighting factors of DEWMA controller in (45) are $\omega_{1}=1-\lambda^{2}=0.2775$ and $\omega_{2}=(1-\lambda)^{2}=0.0225$ accordingly. If metrology delays exist while controlling the process model in (1), then both the actual metrology delay $\left(t^{\prime}\right)$ and the predicted metrology delay $\left(t_{p}\right)$ need to be considered in the control actions of DEWMA and 
RLS-LT, as given in Table 1 respectively.

First, the actual metrology delay is assumed to be $t^{\prime}=1$ and three conditions of the predicted metrology delay are examined: $1\left(t_{p}=t^{\prime}\right.$, correctly estimated $), 0$ ( $t_{p}<t^{\prime}$, underestimated) and 2 ( $t_{p}>t^{\prime}$ overestimated). Fourteen cases of the drift per run are tested, $\delta=-3$ : $:(0.5): 3$. In each experiment, the performance statistics, including mean squared error from target (MSE) and mean, are calculated based on the simulation results of 200 runs each and 200 simulation cycles.

Table 1. DEWMA and RLS-LT control with metrology delays.

\begin{tabular}{|c|c|}
\hline \multicolumn{2}{|r|}{ DEWMA controller } \\
\hline Process model & $y_{t}=\alpha+\beta u_{t-1}+\delta t+\varepsilon_{t}$ \\
\hline $\begin{array}{l}\text { Recursive } \\
\text { algorithms }\end{array}$ & $\begin{aligned} \text { (i) } a_{t} & =\omega_{1} x_{t}+\left(1-\omega_{1}\right)\left(a_{t-1}+D_{t-1}\right) \\
\text { (ii) } D_{t} & =\omega_{2}\left(x_{t}-a_{t-1}\right)+\left(1-\omega_{2}\right) D_{t-1}\end{aligned}$ \\
\hline Control action & $u_{t}=\frac{\tau-a_{t-t^{\prime}}-\left(1+t_{p}\right) D_{t-t^{\prime}}}{b}$ \\
\hline \multicolumn{2}{|r|}{ RLS-LT controller } \\
\hline $\begin{array}{l}\text { Linear trend } \\
\text { model }\end{array}$ & $x_{t}=y_{t}-\beta u_{t-1}=\boldsymbol{\varphi}_{t}^{\mathrm{T}} \boldsymbol{\theta}+\varepsilon_{t}$ \\
\hline $\begin{array}{l}\text { Recursive } \\
\text { algorithms }\end{array}$ & $\begin{array}{l}\text { (i) } \boldsymbol{K}_{t}=\frac{\boldsymbol{P}_{t-1} \boldsymbol{\varphi}_{t}}{\lambda+\boldsymbol{\varphi}_{t}^{\mathrm{T}} \boldsymbol{P}_{t-1} \boldsymbol{\varphi}_{t}} \\
\text { (ii) } \boldsymbol{P}_{t}=\frac{\left(\mathbf{I}-\boldsymbol{K}_{t} \boldsymbol{\varphi}_{t}^{\mathrm{T}}\right) \boldsymbol{P}_{t-1}}{\lambda} \\
\text { (iii) } \hat{\boldsymbol{\theta}}_{t}=\hat{\boldsymbol{\theta}}_{t-1}+\boldsymbol{K}_{t}\left(x_{t}-\boldsymbol{\varphi}_{t}^{\mathrm{T}} \hat{\boldsymbol{\theta}}_{t-1}\right)\end{array}$ \\
\hline Control action & $=\frac{\tau-\hat{\theta}_{1_{t-t^{\prime}}}-\left[1+\left(t-t^{\prime}\right)+t_{p}\right] \hat{\theta}_{2_{t-t^{\prime}}}}{b}$ \\
\hline
\end{tabular}

Table 2 Table 3 show the simulation results returned by using the DEWMA and RLS-LT controllers under the influence of metrology delays and deterministic drifts. On the basis of using a single tuning factor, the RLS-LT controller performs better than the DEWMA controller in these two performance statistics. However, if the weight factors, $\omega_{1}$ and $\omega_{2}$, are individually determined and appropriately chosen, then DEWMA controller can obtain smaller MSE than the RLS-LT controller for this situation (Fan et al., 2002). Apparently from Table 2 Table 3 and Figure 1, if the metrology delay is correctly estimated $\left(t_{p}=t^{\prime}=1\right)$, the mean of the process output is expected to be asymptotically close to target. The mean deviates from target when the metrology delay is incorrectly estimated and/or the drift rate rises. The RLS-LT controller shows good robustness to the drift increase and delay mismatch in MSE. By contrast, the performance of the DEWMA controller in MSE becomes increasingly aggravated as the drift rate increases or decreases away from 0. Several interesting observations for both controllers deserve to report (i) when the metrology delay is correctly estimated $\left(t_{p}=t^{\prime}=1\right)$, in case of $\delta>0$ the deviation of mean is positive, and negative otherwise; (ii) when the metrology delay is underestimated $\left(t_{p}<t^{\prime}\right)$, in case of $\delta>0$ the deviation of mean is positive, and negative otherwise; (iii) when the metrology delay is overestimated $\left(t_{p}>t^{\prime}\right)$, in case of $\delta>0$ the deviation of mean is negative, and positive otherwise.

Table 2. Simulation results with one unit of metrology delay using the DEWMA controller.

\begin{tabular}{|c|c|c|c|c|c|c|}
\hline \multirow{2}{*}{$\delta$} & \multicolumn{2}{|c|}{$\left(t_{p}=0\right)<\left(t^{\prime}=1\right)$} & \multicolumn{2}{c|}{$\left(t_{p}=1\right)=\left(t^{\prime}=1\right)$} & \multicolumn{2}{c|}{$\left(t_{p}=2\right)>\left(t^{\prime}=1\right)$} \\
\cline { 2 - 7 } & MSE & mean & MSE & mean & MSE & mean \\
\hline \hline 3 & 4.376 & 3.662 & 6.551 & 0.863 & 9.459 & -1.936 \\
\hline 2.5 & 3.408 & 3.052 & 4.927 & 0.719 & 6.955 & -1.614 \\
\hline 2 & 2.615 & 2.442 & 3.598 & 0.575 & 4.906 & -1.291 \\
\hline 1.5 & 1.999 & 1.831 & 2.563 & 0.431 & 3.313 & -0.968 \\
\hline 1 & 1.558 & 1.221 & 1.825 & 0.288 & 2.174 & -0.645 \\
\hline 0.5 & 1.293 & 0.611 & 1.381 & 0.144 & 1.490 & -0.323 \\
\hline 0.1 & 1.208 & 0.122 & 1.238 & 0.029 & 1.271 & -0.065 \\
\hline-0.1 & 1.208 & -0.122 & 1.238 & -0.029 & 1.271 & 0.065 \\
\hline-0.5 & 1.291 & -0.610 & 1.379 & -0.144 & 1.488 & 0.323 \\
\hline-1 & 1.554 & -1.221 & 1.821 & -0.287 & 2.170 & 0.645 \\
\hline-1.5 & 1.993 & -1.831 & 2.558 & -0.431 & 3.306 & 0.968 \\
\hline-2 & 2.608 & -2.441 & 3.590 & -0.575 & 4.898 & 1.291 \\
\hline-2.5 & 3.398 & -3.052 & 4.917 & -0.719 & 6.944 & 1.614 \\
\hline-3 & 4.364 & -3.662 & 6.539 & -0.862 & 9.446 & 1.936 \\
\hline
\end{tabular}

Table 3. Simulation results with one unit of metrology delay using the RLS-LT controller.

\begin{tabular}{|c|c|c|c|c|c|c|}
\hline \multirow{2}{*}{$\delta$} & \multicolumn{2}{|c|}{$\left(t_{p}=0\right)<\left(t^{\prime}=1\right)$} & \multicolumn{2}{l|}{$\left(t_{p}=1\right)=\left(t^{\prime}=1\right)$} & \multicolumn{2}{c|}{$\left(t_{p}=2\right)>\left(t^{\prime}=1\right)$} \\
\cline { 2 - 7 } & MSE & mean & MSE & mean & MSE & mean \\
\hline \hline 3 & 1.315 & 3.001 & 1.542 & 0.046 & 2.057 & -2.894 \\
\hline 2.5 & 1.309 & 2.501 & 1.495 & 0.038 & 1.874 & -2.412 \\
\hline 2 & 1.303 & 2.001 & 1.457 & 0.031 & 1.724 & -1.930 \\
\hline 1.5 & 1.299 & 1.500 & 1.427 & 0.023 & 1.608 & -1.447 \\
\hline 1 & 1.296 & 1.000 & 1.406 & 0.015 & 1.525 & -0.965 \\
\hline 0.5 & 1.294 & 0.500 & 1.393 & 0.008 & 1.476 & -0.483 \\
\hline 0.1 & 1.293 & 0.100 & 1.389 & 0.001 & 1.460 & -0.097 \\
\hline-0.1 & 1.293 & -0.100 & 1.390 & -0.002 & 1.461 & 0.096 \\
\hline-0.5 & 1.294 & -0.500 & 1.394 & -0.008 & 1.477 & 0.482 \\
\hline-1 & 1.296 & -1.000 & 1.408 & -0.016 & 1.528 & 0.964 \\
\hline-1.5 & 1.299 & -1.500 & 1.430 & -0.023 & 1.613 & 1.446 \\
\hline-2 & 1.304 & -2.001 & 1.461 & -0.031 & 1.731 & 1.929 \\
\hline-2.5 & 1.309 & -2.501 & 1.501 & -0.039 & 1.882 & 2.411 \\
\hline-3 & 1.316 & -3.001 & 1.549 & -0.046 & 2.066 & 2.893 \\
\hline
\end{tabular}


To reconfirm the conjecture aforementioned, an extended simulation is conducted. The actual metrology delay is assumed to be $2\left(t^{\prime}=2\right)$, and 5 conditions of the predicted metrology delay are investigated: $0\left(t_{p}<t^{\prime}\right), 1$ $\left(t_{p}<t^{\prime}\right), 2\left(t_{p}=t^{\prime}\right), 3\left(t_{p}>t^{\prime}\right)$ and $4\left(t_{p}>t^{\prime}\right)$. The simulation results are listed in Table 4-Table 5 and illustrated in Figure 2. Clearly from tables, the more badly estimated the metrology delay, the more considerable deviation of mean from target, especially for instances of $t_{p}=0$ in DEWMA. Overall, the results of Table 4 Table 5, showing that the RLS-LT controller produces more stable process outputs than the DEWMA controller (see MSE in Table 4 Table 5). More importantly, the previously mentioned 3 observations are still held. The simulation results concur partly with the fact reported by Wang et al. (2005) that RLS-type estimates responded to process changes faster than EWMA-type estimates.
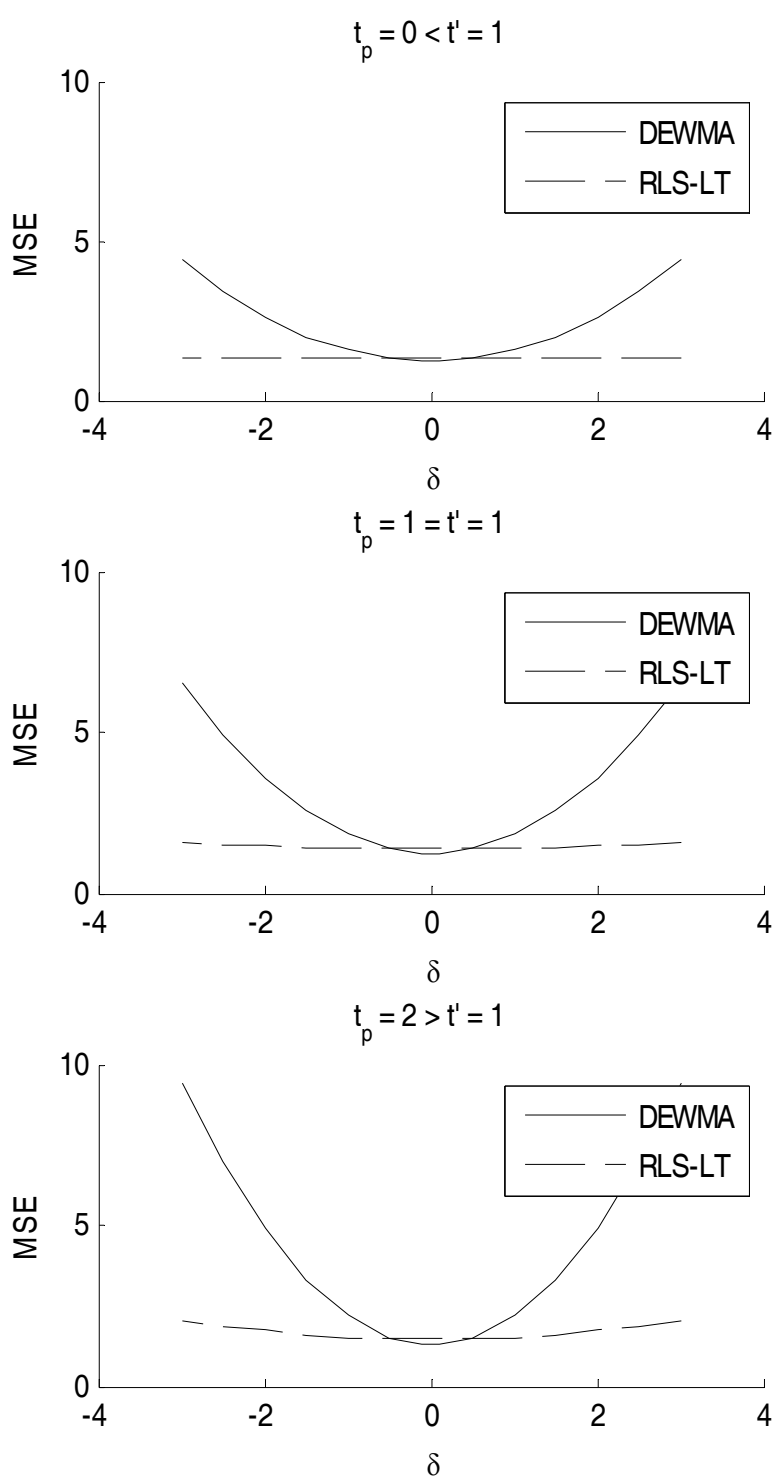

Figure 1. MSE for one unit of metrology delay using the DEWMA controller.
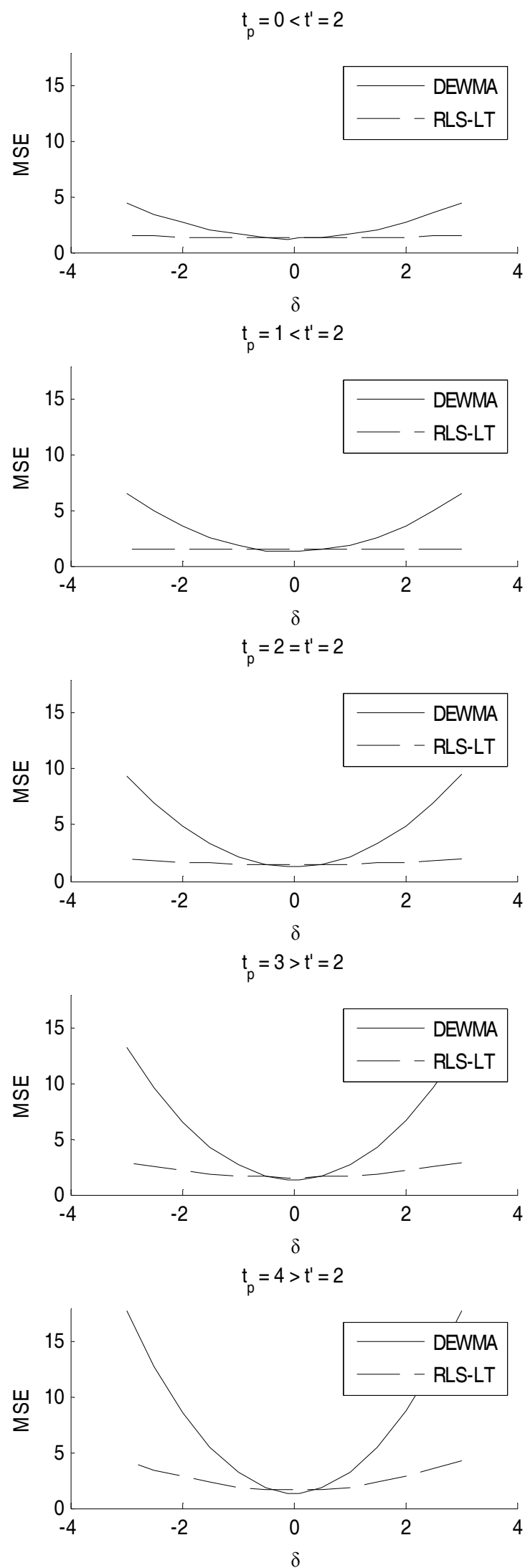

Figure 2. MSE for one unit of metrology delay using the RLS-LT controller. 
Table 4. Extended simulation results with two units of metrology delay using the DEWMA controller.

\begin{tabular}{|c|c|c|c|c|c|c|c|c|c|c|}
\hline \multirow{2}{*}{$\delta$} & \multicolumn{2}{|c|}{$\begin{array}{c}\text { Underestimated: } \\
\left(t_{p}=0\right)<\left(t^{\prime}=2\right)\end{array}$} & \multicolumn{2}{c|}{$\begin{array}{c}\text { Underestimated: } \\
\left(t_{p}=1\right)<\left(t^{\prime}=2\right)\end{array}$} & \multicolumn{2}{c|}{$\begin{array}{c}\text { Correctly Estimated: } \\
\left(t_{p}=2\right)=\left(t^{\prime}=2\right)\end{array}$} & \multicolumn{2}{c|}{$\begin{array}{c}\text { Overestimated: } \\
\left(t_{p}=3\right)>\left(t^{\prime}=2\right)\end{array}$} & \multicolumn{2}{c|}{$\begin{array}{c}\text { Overestimated: } \\
\left(t_{p}=4\right)>\left(t^{\prime}=2\right)\end{array}$} \\
\cline { 2 - 13 } & MSE & mean & MSE & mean & MSE & mean & MSE & mean & MSE & mean \\
\hline \hline 3 & 4.4588 & 6.6429 & 6.5443 & 3.8581 & 9.4395 & 1.0743 & 13.181 & -1.7079 & 17.820 & -4.4876 \\
\hline 2.5 & 3.4670 & 5.5358 & 4.9237 & 3.2151 & 6.9435 & 0.8953 & 9.5513 & -1.4233 & 12.784 & -3.7397 \\
\hline 2 & 2.6552 & 4.4287 & 3.5975 & 2.5721 & 4.9010 & 0.7162 & 6.5816 & -1.1386 & 8.6625 & -2.9918 \\
\hline 1.5 & 2.0234 & 3.3215 & 2.5657 & 1.9291 & 3.3120 & 0.5372 & 4.2714 & -0.8540 & 5.4567 & -2.2439 \\
\hline 1 & 1.5717 & 2.2144 & 1.8282 & 1.2861 & 2.1765 & 0.3581 & 2.6206 & -0.5693 & 3.1663 & -1.4960 \\
\hline 0.5 & 1.3000 & 1.1073 & 1.3850 & 0.6431 & 1.4945 & 0.1791 & 1.6294 & -0.2847 & 1.7912 & -0.7481 \\
\hline 0.1 & 1.2122 & 0.2216 & 1.2423 & 0.1287 & 1.2754 & 0.0359 & 1.3113 & -0.0570 & 1.3502 & -0.1497 \\
\hline-0.1 & 1.2115 & -0.2212 & 1.2417 & -0.1285 & 1.2747 & -0.0357 & 1.3106 & 0.0569 & 1.3493 & 0.1494 \\
\hline-0.5 & 1.2966 & -1.1069 & 1.3816 & -0.6429 & 1.4909 & -0.1790 & 1.6256 & 0.2846 & 1.7870 & 0.7478 \\
\hline-1 & 1.5649 & -2.2140 & 1.8214 & -1.2859 & 2.1694 & -0.3580 & 2.6129 & 0.5692 & 3.1578 & 1.4957 \\
\hline-1.5 & 2.0133 & -3.3211 & 2.5556 & -1.9289 & 3.3014 & -0.5371 & 4.2598 & 0.8539 & 5.4440 & 2.2436 \\
\hline-2 & 2.6417 & -4.4282 & 3.5841 & -2.5719 & 4.8868 & -0.7161 & 6.5662 & 1.1385 & 8.6454 & 2.9915 \\
\hline-2.5 & 3.4501 & -5.5353 & 4.9069 & -3.2149 & 6.9258 & -0.8952 & 9.5321 & 1.4232 & 12.762 & 3.7394 \\
\hline-3 & 4.4385 & -6.6425 & 6.5241 & -3.8579 & 9.4183 & -1.0742 & 13.158 & 1.7078 & 17.794 & 4.4873 \\
\hline
\end{tabular}

Table 5. Extended simulation results with two units of metrology delay using the RLS-LT controller.

\begin{tabular}{|c|c|c|c|c|c|c|c|c|c|c|}
\hline \multirow{2}{*}{$\delta$} & \multicolumn{2}{|c|}{$\begin{array}{c}\text { Underestimated: } \\
\left(t_{p}=0\right)<\left(t^{\prime}=2\right)\end{array}$} & \multicolumn{2}{c|}{$\begin{array}{c}\text { Underestimated: } \\
\left(t_{p}=1\right)<\left(t^{\prime}=2\right)\end{array}$} & \multicolumn{2}{c|}{$\begin{array}{c}\text { Correctly Estimated: } \\
\left(t_{p}=2\right)=\left(t^{\prime}=2\right)\end{array}$} & \multicolumn{2}{c|}{$\begin{array}{c}\text { Overestimated: } \\
\left(t_{p}=3\right)>\left(t^{\prime}=2\right)\end{array}$} & \multicolumn{2}{c|}{$\begin{array}{c}\text { Overestimated: } \\
\left(t_{p}=4\right)>\left(t^{\prime}=2\right)\end{array}$} \\
\cline { 2 - 12 } & MSE & mean & MSE & mean & MSE & mean & MSE & mean & MSE & mean \\
\hline \hline 3 & 1.4751 & 5.9635 & 1.5241 & 3.0237 & 1.9531 & 0.0984 & 2.8583 & -2.8120 & 4.3265 & -5.7076 \\
\hline 2.5 & 1.4238 & 4.9697 & 1.4865 & 2.5198 & 1.8065 & 0.0820 & 2.4550 & -2.3435 & 3.4941 & -4.7566 \\
\hline 2 & 1.3818 & 3.9758 & 1.4557 & 2.0158 & 1.6865 & 0.0655 & 2.1247 & -1.8750 & 2.8123 & -3.8055 \\
\hline 1.5 & 1.3490 & 2.9819 & 1.4318 & 1.5119 & 1.5931 & 0.0491 & 1.8675 & -1.4064 & 2.2812 & -2.8545 \\
\hline 1 & 1.3255 & 1.9880 & 1.4149 & 1.0079 & 1.5265 & 0.0326 & 1.6833 & -0.9379 & 1.9007 & -1.9035 \\
\hline 0.5 & 1.3112 & 0.9941 & 1.4048 & 0.5040 & 1.4865 & 0.0161 & 1.5723 & -0.4694 & 1.6709 & -0.9525 \\
\hline 0.1 & 1.3065 & 0.1990 & 1.4017 & 0.1008 & 1.4737 & 0.0030 & 1.5360 & -0.0945 & 1.5954 & -0.1916 \\
\hline-0.1 & 1.3063 & -0.1986 & 1.4018 & -0.1007 & 1.4737 & -0.0036 & 1.5354 & 0.0929 & 1.5939 & 0.1888 \\
\hline-0.5 & 1.3104 & -0.9937 & 1.4053 & -0.5039 & 1.4864 & -0.0168 & 1.5693 & 0.4677 & 1.6631 & 0.9496 \\
\hline-1 & 1.3239 & -1.9876 & 1.4160 & -1.0078 & 1.5264 & -0.0332 & 1.6775 & 0.9362 & 1.8852 & 1.9006 \\
\hline-1.5 & 1.3467 & -2.9815 & 1.4335 & -1.5118 & 1.5931 & -0.0497 & 1.8587 & 1.4048 & 2.2579 & 2.8517 \\
\hline-2 & 1.3787 & -3.9753 & 1.4579 & -2.0157 & 1.6864 & -0.0661 & 2.1130 & 1.8733 & 2.7813 & 3.8027 \\
\hline-2.5 & 1.4199 & -4.9692 & 1.4892 & -2.5197 & 1.8064 & -0.0826 & 2.4404 & 2.3418 & 3.4553 & 4.7537 \\
\hline-3 & 1.4704 & -5.9631 & 1.5274 & -3.0236 & 1.9530 & -0.0991 & 2.8409 & 2.8104 & 4.2800 & 5.7048 \\
\hline
\end{tabular}

\subsection{Property of DEWMA and RLS-LT with Metrol- ogy Delays}

In this section, mathematical derivations of the deviation from target will be presented as the metrology delay is taken into account. If the DEWMA controller is used in (1) without metrology delays, then the estimate of metrology delay $\left(t_{p}\right)$ is introduced to the process model in the following way:

$$
\begin{aligned}
y_{t} & =\alpha+\beta u_{t-1}+\delta t+\varepsilon_{t} \\
& =\alpha+\beta\left(\frac{\tau-a_{t-1}-\left(1+t_{p}\right) D_{t-1}}{b}\right)+\delta t+\varepsilon_{t} \\
& =\alpha+\xi \tau-\xi a_{t-1}-\xi\left(1+t_{p}\right) D_{t-1}+\delta t+\varepsilon_{t},
\end{aligned}
$$

where $\xi=\beta / b$ and $\beta$ is assumed known or closely estimated; that is, $\xi \cong 1$. If the weighting factors are chosen adequately in the stable region, as $t \rightarrow \infty$ the ex- 
pected value of $y_{t}$ is

$$
\begin{aligned}
E\left(y_{t}\right) & =E\left(\alpha+\tau-a_{t-1}-\left(1+t_{p}\right) D_{t-1}+\delta t\right) \\
& =E\left(\tau-t_{p} D_{t-1}+\alpha+\delta t-a_{t-1}-D_{t-1}\right) \\
& =\tau-t_{p} \delta
\end{aligned}
$$

since $D_{t-1}$ is the estimate for $\delta$ and $a_{t-1}+D_{t-1}$ is the estimate for $\alpha+\delta t$. There are six possible cases:

(A-1) if $\delta>0$ and $t_{p}>0$, then $E\left(y_{t}\right)<\tau$;

(A-2) if $\delta>0$ and $t_{p}=0$, then $E\left(y_{t}\right)=\tau$;

(A-3) if $\delta>0$ and $t_{p}<0$, then $E\left(y_{t}\right)>\tau$;

(A-4) if $\delta<0$ and $t_{p}>0$, then $E\left(y_{t}\right)>\tau$;

(A-5) if $\delta<0$ and $t_{p}=0$, then $E\left(y_{t}\right)=\tau$;

(A-6) if $\delta<0$ and $t_{p}<0$, then $E\left(y_{t}\right)<\tau$

In practice, however, the three cases with $t_{p}<0$ are not applicable in the R2R problem. Next, the actual metrology delay $\left(t^{\prime}\right)$ is put into effect, leading to the following lemma.

Lemma 1: If metrology delays occur during controlling the process model in (1), then the DEWMA controller yields the asymptotic output in the following six cases:

$(B-1)$ if $\delta>0$ and $t_{p}>t^{\prime}$, then $E\left(y_{t}\right)<\tau$;

$(B-2)$ if $\delta>0$ and $t_{p}=t^{\prime}$, then $E\left(y_{t}\right)=\tau$;

$(B-3)$ if $\delta>0$ and $t_{p}<t^{\prime}$, then $E\left(y_{t}\right)>\tau$;

$(B-4)$ if $\delta<0$ and $t_{p}>t^{\prime}$, then $E\left(y_{t}\right)>\tau$;

$(B-5)$ if $\delta<0$ and $t_{p}=t^{\prime}$, then $E\left(y_{t}\right)=\tau$;

$(B-6)$ if $\delta<0$ and $t_{p}<t^{\prime}$, then $E\left(y_{t}\right)<\tau$.

Proof: See Appendix I. A similar lemma can be obtained for the RLS-LT controller as follows.

Lemma 2: If metrology delays occur during controlling the process model in (1), then the RLS-LT controller yields the asymptotic output in the following six cases:

$(C-1)$ if $\delta>0$ and $t_{p}>t^{\prime}$, then $E\left(y_{t}\right)<\tau$;

$(C-2)$ if $\delta>0$ and $t_{p}=t^{\prime}$, then $E\left(y_{t}\right)=\tau$;

$(C-3)$ if $\delta>0$ and $t_{p}<t^{\prime}$, then $E\left(y_{t}\right)>\tau$;

$(C-4)$ if $\delta<0$ and $t_{p}>t^{\prime}$, then $E\left(y_{t}\right)>\tau$;

$(C-5)$ if $\delta<0$ and $t_{p}=t^{\prime}$, then $E\left(y_{t}\right)=\tau$;

$(C-6)$ if $\delta<0$ and $t_{p}<t^{\prime}$, then $E\left(y_{t}\right)<\tau$.

Proof: See Appendix II.

No matter the DEWMA or RLS-LT controller is used, the derived mathematical properties can provide these two controllers with useful guidelines for the prediction of the actual metrology delay for run to run. At the outset of R2R processes, the actual metrology delay can be deemed as known and constant, but might vary as time elapses due to unstable work-in-process (WIP), maintenance operation, tool-wearing and other unpredictable intervention. Thus, the pattern of metrology delays could be random, lumpy, increasing, decreasing, or even cyclic.

\section{PROPOSED RLS-LT CONTROLLER WITH METROLOGY DELAYS USING RUNS TEST}

\subsection{Runs Test}

In many situations we want to know whether we can conclude that a series or items or runs is random (because the error is white). An important example is the sample of data available for some statistical analysis. A basic assumption underlying procedures for statistical inference is that the inference is based on a white noise sequence (Daniel, 1990). If the randomness of an error is suspect, we want to decide whether the run is random before we proceed with the analysis.

Procedures for investigating randomness are based on the number and nature of the runs present in the data of interest. A run is defined as a sequence of like events, items, or symbols that is preceded and followed by an event, items, or symbols in a run is referred to as its length (Siegel and Castellan, 1988). We doubt the randomness of a series when there appear to be either too many or too few runs. Now, let us consider the onesample runs test. This procedure helps us decide whether a sequence of events, items, or symbols is the result of a random process.

\subsubsection{Assumptions}

The data available for analysis consist of a sequence of observations, recorded in the order of their occurrence, which we can categorize into two mutually exclusive types. We let $\mathrm{n}=$ the total sample size, $n_{1}=$ the number of observations of one type, and $n_{2}=$ the number of observations of the other type $\left(n_{1} \leq n_{2}\right)$.

\subsubsection{Hypotheses}

(Two-sided)

$H_{0}$ : The pattern of occurrence of the two types of observation is determined by arandom process (sequence is random. $H_{a}$ : The pattern of occurrence is nonrandom (sequence is nonrandom).

\subsubsection{Test Statistic}

The test statistic is $r$, the total number of runs.

\subsubsection{Decision Rule}

A. Small samples $\left(n_{1} \leq n_{2} \leq 10\right)$

Table runs test of the appendix gives the accumulated probability of runs test $(P(R \leq r))$. The level of significance is given (generally, $\alpha=0.01$ or $\alpha=0.05$ ), If the table value has satisfied: $\alpha / 2 \leq$ table value $\leq(1-\alpha / 2)$, $H_{0}$ can not be rejected; if the table value is either more than $(1-\alpha / 2)$ or less than $\alpha / 2, H_{0}$ can be rejected. 
B. Large samples $\left(n_{1}>10\right.$ or $\left.n_{2}>10\right)$

If either $n_{1}$ or $n_{2}$ is larger than 10, appendix table runs test can not be used. For such large samples, a good approximation to the sampling distribution of $r$ is the normal distribution with mean

$$
E(r)=1+\frac{2 n_{1} n_{2}}{n_{1}+n_{2}}
$$

and standard deviation

$$
\operatorname{Var}(r)=\frac{2 n_{1} n_{2}\left(2 n_{1} n_{2}-n_{1}-n_{2}\right)}{\left(n_{1}+n_{2}\right)^{2}\left(n_{1}+n_{2}-1\right)}
$$

Therefore, when either $n_{1}$ or $n_{2}$ is greater then 10 , $H_{0}$ can be tested by

$$
Z^{*}=\frac{r-E(r)}{\sqrt{\operatorname{Var}(r)}}
$$

The level of significance is given $(\alpha)$, and if $\left|Z^{*}\right| \geq Z_{\frac{\alpha}{2}}, \quad H_{0}$ can be rejected; if not, $H_{0}$ can not be rejected. ${ }^{\frac{a}{2}}$ Especially, when $n_{1}=n_{2}=n / 2, E(r)=1+n / 2$, $\operatorname{Var}(r)=n(n-2) / 4(n-1)$.

\subsection{RLS-LT Controller Using Runs Test}

Before using the proposed modified RLS-LT controller, the pre-procedures are needed to obtain the optimal discounting factor, the start checking point (the first run after the process transition).

It is assumed that the level of significance $(\alpha)$ is given. According to the last successive runs which have the same number of predicted metrology delay, calculating their median and we can categorize the samples into two mutually exclusive types (more than or less than). Let $n=$ the total sample size, $n_{1}=$ the number of type one observations, $n_{2}=$ the number of type two observations $\left(n_{1} \leq n_{2}\right)$, and $r$ is the total number of runs. Then, putting $n, n_{1}, n_{2}, r$ to use, the runs test can be used. If $n_{1} \leq n_{2} \leq 10$ (small samples), the runs test is used to look for the accumulated probability of appendix table runs test; if $n_{1}>10$ or $n_{2}>10$ (large samples), the random sequence assumption can be tested by $\mathrm{Z}$-value.

If $H_{0}$ could not be rejected, the predicted metrology delay is unchanged; if $H_{0}$ is rejected, it means that the process is out of control. The assignable cause could be due to the incorrect prediction of metrology delay which is encountered in real-case. Based on the fundamental insight of expected deviation of the process from target, the prediction of metrology delay can be adaptively adjusted.

When the metrology delay is underestimated $\left(t_{p}<\right.$ $t^{\prime}$ ), the controlled process output drifts upward from $\tau$ as $\delta$ is positive and downward from $\tau$ as $\delta$ is negative.
When the metrology delay is overestimated $\left(t_{p}>t^{\prime}\right)$, the controlled process output drifts downward from $\tau$ as $\delta$ is positive and upward from $\tau$ as $\delta$ is negative. Therefore, when the controlled process output is out of control, if the estimate of drift is positive, the prediction of metrology delay is increasing $(+1)$ if the out of control process output is higher than $\tau$, and decreasing $(-1)$ if the out of control process output is lower than $\tau$; if the out of control process output is lower than $\tau$. If the predicted metrology delay is changed, the check point becomes the time index plus 5 because runs test needs at least five observations in the small sample size.

The flowchart of the modified RLS-LT controller is shown in Figure 3 and the procedure of the modified RLS-LT controller is given as follows:

Step 1: Provide the simple first-order linear model with a set of initial parameter estimates from an offline DOE/RSM work.

Step 2: Under different constituting of metrology delay and gain mismatch, the pre-procedures are done to obtain:

(1) the optimal discounting factor,

(2) the start checking point.

Step 3: The level of significance is given, $\alpha$. Starting the discrete time index with $\mathrm{t}=1$, and predicted metrology delay $=0$.

Step 4: There are time-varying metrology delays in the process. Run the RLS-LT controller (section 3.1.3). If the time index is reaching the checking point, then go to step 5 , otherwise go to step 10 .

Step 5: Runs test mechanism is used. $\mathrm{n}=$ the total sample size that the successive runs have the same number of predicted metrology delay. If $\mathrm{n}$ is bigger than 5 , then go to step 6 , otherwise go to step 10 .

Step 6: The hypotheses are $H_{0}$ : sequence is random, $H_{a}$ : sequence is nonrandom. $\mathrm{n}_{1}$ is the number of observations ( $>$ median), and $n_{2}$ is the number of observations ( $<$ median). If $\mathrm{n}_{1}>10$ or $\mathrm{n}_{2}>10$, then go to step 8, otherwise go to step 7 .

Step 7: Small samples: using $\mathrm{n}_{1}, \mathrm{n}_{2}$, and $\mathrm{r}$ (the total number of runs) to look for the value of runs test table. If $\alpha / 2 \leq$ table value $\leq(1-\alpha / 2)$, then go to step 9, otherwise go to step 10 .

Step 8: Large samples: approximation to the sampling distribution of $\mathrm{r}$ is the normal distribution with $E(r)$ and $\operatorname{Var}(r) . H_{0}$ can be tested by $Z^{*}=r$ $-E(r) / \sqrt{\operatorname{Var}(r)}$. If $\left|Z^{*}\right| \geq Z_{\alpha / 2}$, then go to step 9, otherwise go to step 10 .

Step 9: According to the drift estimate, the latest process output and $\tau$, regulating the predicted metrology delay. Update the checking point.

Step 10: The recipe is renovated as $\mu_{t}$.

Step 11: Set $t \leftarrow t+1$. Return to step 4 . 
In Figure 3, a set of initial parameter estimates obtained from an off-line experiment are provided with the process engineer to initiate the control task. A comprehensive pre-control simulation study is conducted to gain knowledge about the optimal discounting factor, the duration of transient period, and the parameters by simulating the following model:

$$
\tilde{Y}_{t}=\hat{\alpha}+\hat{\beta} u_{t-1}+\hat{\delta} t+\hat{\varepsilon}_{t}
$$

$\tilde{Y}_{t}$ denotes the "simulated" process output under the control of RLS-LT; $\hat{\alpha}, \hat{\beta}, \hat{\delta}$ are the parameter estimates of the process model obtained from an off-line DOE or pilot plant data. The error estimate $\hat{\varepsilon}_{t}$ is assumed to approximately follow $N(0, \sqrt{M S E})$, where $M S E$ is the mean squares error from the analysis of variance (ANOVA). First, the optimal discounting factor is determined by plotting $\lambda$ versus mean squared error (MSE) and mean process output. The plot of $\lambda$ versus MSE shows that the MSE of the RLS-LT controller is very sensitive when $\lambda$ is small, while the plot of $\lambda$ versus process mean suggests that the controller is robust in converging to target. In this paper, the minimum $\lambda$ where MSE becomes less sensitive to $\lambda$ and process mean converges to target value is chosen as the optimal discounting factor. Second, 30 time periods are tested, $t_{1} \sim t_{100}, t_{2} \sim t_{100}, \cdots, t_{30} \sim t_{100}$, under different delays in pairs $\left(t^{\prime}=t_{p}=0,1, \cdots, 5\right)$ to decide appropriate transient period. The starting check point after transient is the run when MSE stabilizes.

After the deviation from target which is caused by incorrect prediction of metrology delay has been proven, this thesis modifies the RLS-LT controller and presents a method to control the time-varying metrology delay process. The method is one kind of nonparametric statistics. The capabilities of the proposed controller will be tested through a variety of simulations in Section 5 .

\section{EXPERIMENTAL STUDY OF THE MODI- FIED RLS-LT CONTROLLER WITH TIME-VARYING METROLOGY DELAYS}

The simulated equipment model (referring to the example discussed in Wang et al., 2005) treated as the true production system is given in the Table 6 . It is assumed that the target value $\tau=0$, the process offset $\alpha=0$, the process gain $\beta=4$, and the process is assumed to be at steady state initially. In each experiment, the performance statistics, MSE and mean, are calculated based on the simulation results of 100 runs each and 1000 simulation cycles. Process drift of $\delta=1$ per run occurs at run 5, and zero-mean white noise with variance $0.7^{2}$ is added to the process measurements starting from run 5 . In this paper, three kinds of ap- proximate models (model mismatch) are considered. Two of them are model uncertainty by setting $\beta / b=$ $4 / 3$ (33\% uncertainty) and $\beta / b=4 / 5$ (20\% uncertainty), respectively, and the other one is without model mismatch $(\beta / b=4 / 4,0 \%$ uncertainty). The three approximate models are stared with initial process offset estimate $\hat{\alpha}=0$, and initial process drift estimate $\hat{\delta}_{0}=0$.

Table 6. The experimental model and the approximate models of the experiment.

\begin{tabular}{|c|c|}
\hline Experimental model & Approximate models \\
\hline $\begin{array}{c}Y_{t}=\alpha+\beta u_{t-1}+\delta t+\varepsilon_{t} \\
\left(X_{t}=Y_{t}-\beta u_{t-1}=\alpha+\delta t+\varepsilon_{t}\right)\end{array}$ & $\begin{array}{c}\hat{Y}_{t}=\hat{\alpha}+\hat{\beta} u_{t-1}+\hat{\delta} t \\
\left(\hat{X}_{t}=\hat{Y}_{t}-\hat{\beta} u_{t-1}=\hat{\alpha}+\hat{\delta} t\right)\end{array}$ \\
\hline \multirow{3}{*}{$\begin{array}{c}Y_{t}=0+4 \cdot u_{t-1}+\delta t+\varepsilon_{t} \\
X_{t}=Y_{t}-4 \cdot u_{t-1}=0+\delta t+\varepsilon_{t} \\
\text { when } t \geq 5, \quad t=5,6, \cdots \\
\delta=1, \\
\varepsilon_{t} \sim N\left(0,0.7^{2}\right)\end{array}$} & $\begin{array}{c}\hat{Y}_{t}=\hat{\alpha}_{t}+3 \cdot u_{t-1}+\hat{\delta} t \\
\left(\hat{X}_{t}=\hat{Y}_{t}-3 \cdot u_{t-1}=\hat{\alpha}_{t}+\hat{\delta} t\right)\end{array}$ \\
\hline & $\begin{array}{c}\hat{Y}_{t}=\hat{\alpha}_{t}+4 \cdot u_{t-1}+\hat{\delta} t \\
\left(\hat{X}_{t}=\hat{Y}_{t}-4 \cdot u_{t-1}=\hat{\alpha}_{t}+\hat{\delta} t\right)\end{array}$ \\
\hline & $\begin{array}{c}\hat{Y}_{t}=\hat{\alpha}_{t}+5 \cdot u_{t-1}+\hat{\delta} t \\
\left(\hat{X}_{t}=\hat{Y}_{t}-5 \cdot u_{t-1}=\hat{\alpha}_{t}+\hat{\delta} t\right)\end{array}$ \\
\hline
\end{tabular}

For the purpose of illustrating the capabilities of the proposed controller, preliminary work needs to be done in first to obtain (i) the optimal discounting factors; (ii) the starting check point. First, the optimal discounting factors of the controllers need to be chosen: $\omega$ for the EWMA controller, and $\lambda$ for the RLS-LT controller. Therefore, a one-dimensional search is done to find the optimal discounting factors. Based on the searching results, optimal discounting factors are set to perform simulation ( $\omega=0.7$ and $\lambda=0.8)$. Second, the starting check point is $(20+5)$ run because runs test needs at least five observations in the small sample size.

Suppose that the process is operated under a lager batch, 500 runs. The profile of time-varying metrology delays is shown in Figure 4 and described as follows: 0 for runs 1-50, 1 for runs 51-100, 2 for runs 101-150, 3 for runs 151-200, 4 for runs 201-250, 3 for runs 251-300, 2 for runs 301-350, 1 for runs 351-400 and 0 for runs 401-500. The change pattern looks like a cycle, emulating the trouble accumulation, detection and then recovery. The simulation results are tabulated in Table 7 . The modified RLS-LT algorithm exhibits excellent performance in reducing the output variability and deviation from target as compared to using a fixed predicted metrology delay in the RLS-LT controller. Another interesting observation deserves to mention is that the underestimation case $(b=3)$ for controllers $(1-8)$ outperforms the overestimation case $(b=5)$ in almost every performance measurements. 


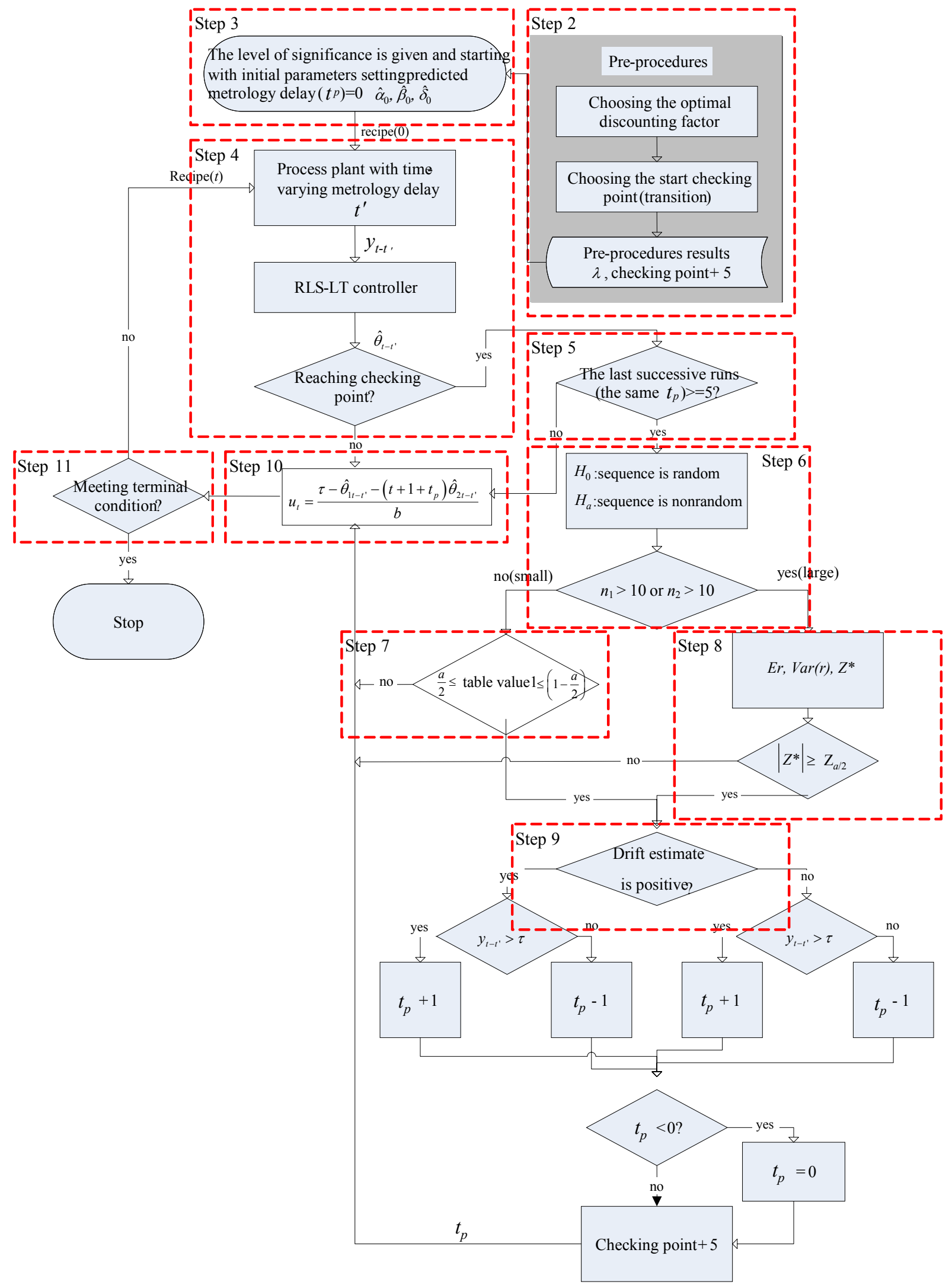

Figure 3. The flowchart of the proposed method. 


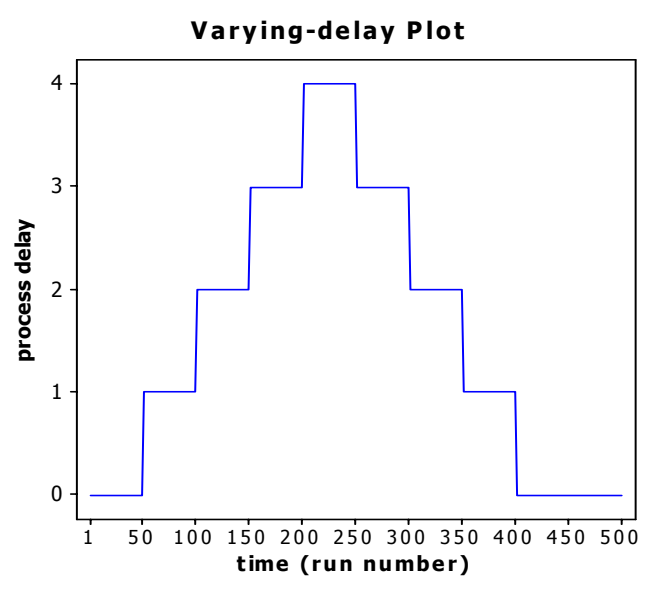

Figure 4. time-varying delay profile in the simulation.

The level of significance $\alpha$ is set to 0.05 . Under different model uncertainty, the control performances are shown in Table 7. It shows that the simulation results under different model mismatches. Controllers (1-6) are the original RLS-LT controller, proposed by Wang et al. (2005), using a fixed number of predicted delay, 0 to $5,\left(t_{p} \in(0,1,2,3,4,5)\right)$ respectively. The simple EWMA controller (controller 7) is merely treated as the benchmark. The weight of $\omega$ is set to 0.7 . The weightselection process is very similar to the one for the RLSLT controller in Section 4. For more details, please refer also to Lo (2007).

In Table 7, it can be seen that RLS_LT controller using runs test is well performance on these two performance measures. According to Table 7, it clearly is shown that using runs test successfully helps the RLSLT controller trace the time-varying metrology delays, thus generating better control outputs (i.e., smaller MSE, and process mean closer to target) than the controllers of simple EWMA and original RLS-LT. It is worth to note again that the original RLS-LT controller is specifically designed for dealing with the constant, known metrology delay cases, so the EWMA and original RLS-LT results in Table 7 are only used for the calibration purpose.

Table 7. Results of simulations (500 runs).

\begin{tabular}{|c|c|c|c|c|c|c|}
\hline & \multicolumn{2}{|c|}{$b=3$} & \multicolumn{2}{c|}{$b=4$} & \multicolumn{2}{c|}{$b=5$} \\
\cline { 2 - 7 } & MSE & mean & MSE & mean & MSE & mean \\
\hline \hline (1) $t_{p}=0$ & 1.75 & 1.21 & 2.43 & 1.61 & 3.39 & 2.01 \\
\hline (2) $t_{p}=1$ & 1.77 & 0.47 & 2.43 & 0.63 & 3.39 & 0.78 \\
\hline (3) $t_{p}=2$ & 1.82 & -0.26 & 2.46 & -0.35 & 3.43 & -0.44 \\
\hline (4) $t_{p}=3$ & 1.88 & -0.99 & 2.52 & -1.33 & 3.53 & -1.68 \\
\hline (5) $t_{p}=4$ & 1.96 & -1.72 & 2.61 & -2.32 & 3.67 & -2.91 \\
\hline (6) $t_{p}=5$ & 2.06 & -2.46 & 2.73 & -3.30 & 3.86 & -4.15 \\
\hline (7) EWMA & 2.03 & 2.264 & 2.64 & 3.01 & 3.61 & 3.76 \\
\hline (8) Runs test & 1.63 & -0.04 & 1.68 & -0.09 & 1.83 & -0.07 \\
\hline
\end{tabular}

Actual delay V.S. predicted delay using runs test $(b=3)$

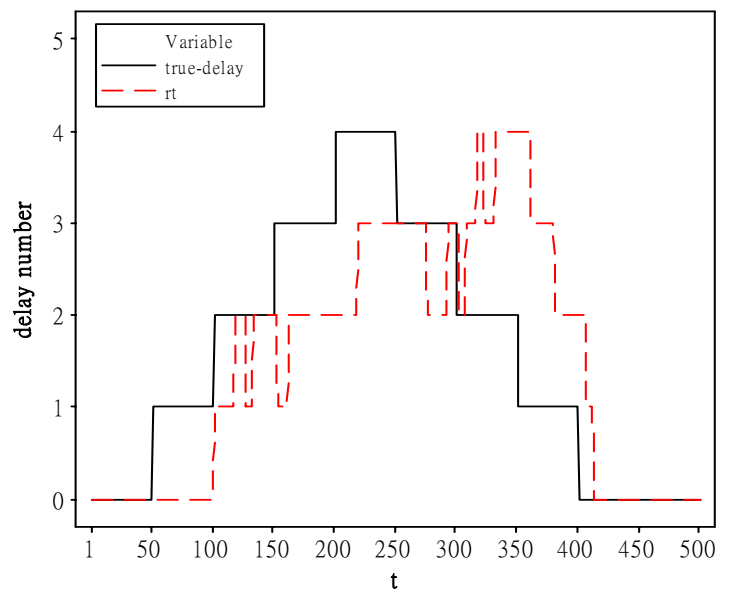

Actual delay V.S.predicted delay using runs test $(b=4)$

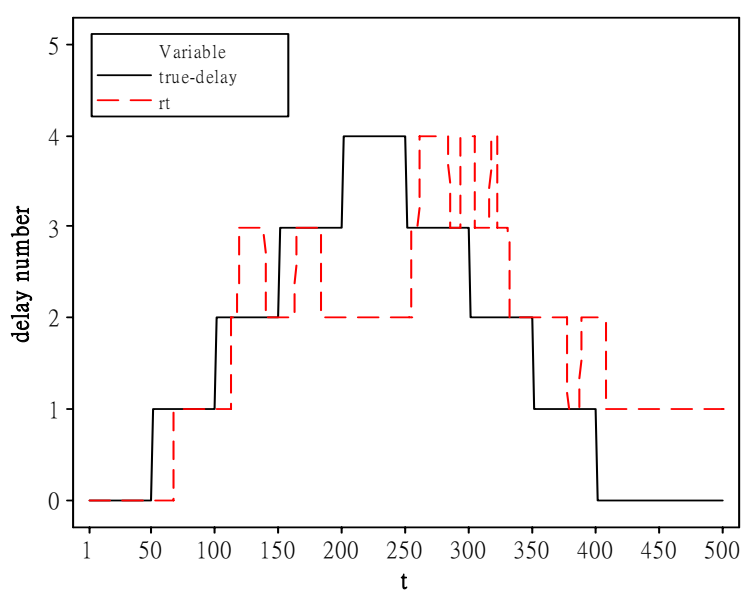

Actual delay V.S. predicted delay using runs test $(b=5)$

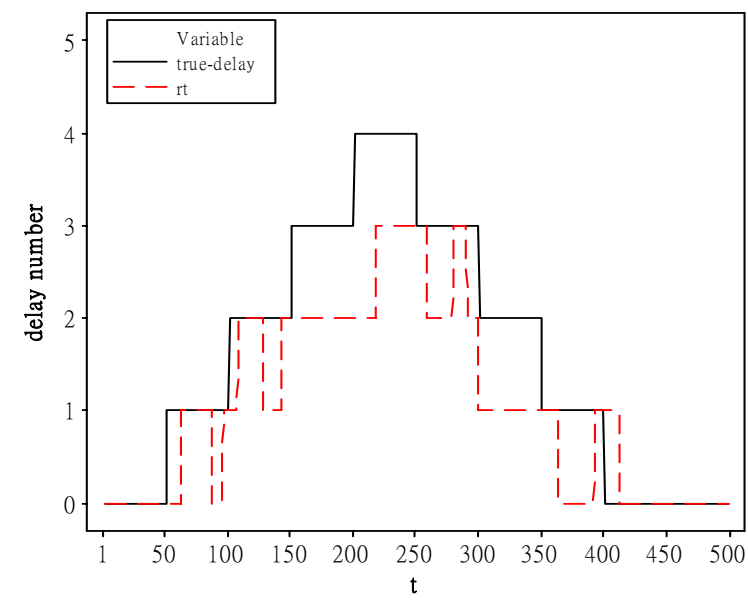

Figure 5. Predicted delay profile generated by using the RLS-LT controller when $b=3,4$ and 5 .

To better illustrate the simulation outcomes, Figure 5 shows the predicted metrology delays generated by using the RLS-LT controller as $b=3,4,5$. The dotted line in the figure represents the predicted profile of the runs test. For example, a step change of metrology delay 
occurs at run 50 and the predicted metrology delay lags behind, like committing a type-II error. Before the actual delay changes from 3 down to 2 around run 290, the delay is adversely forecasted, like committing a type-I error, but is quickly amended at run 300. Apparently, only the modified RLS-LT controller is able to steadily maintain the process output close to target $(\tau=0)$ subject to time-varying metrology delays in Table 7 . For the remaining cases, $\mathrm{b}=3,5$, interested readers can refer to Lo (2007) for detailed simulation results.

\section{CONCLUSION}

Run-to-Run control technology has received tremendous interests in semiconductor manufacturing where EWMA, DEWMA, and RLS estimation are widely recognized methods. It is often the case that a delay exists en route from the process measurement to the process run, called the metrology delay. To address the timevarying metrology delay that could realistically occur in practice, the mathematical property of the DEWMA control output, in terms of the EWMA and RLS prediction methods, is presented. Then, a new time update scheme to the recursive least squares-linear trend (RLSLT) controller, combined with runs test, is proposed in an attempt to dynamically predict the metrology delays. Simulated processes subject to a time-varying metrology delay scenario are used as a test-bed to assess the proposed controller. It has been demonstrated from the simulation results that the modified RLS-LT controller yields the process output more accurately on target with smaller MSE than the original RLS-LT controller that can only deal with constant metrology delays. The proposed RLS-LT controller proves to be a potential tool for solving the time-varying metrology delays in R2R practice.

\section{REFERENCES}

Butler, S. W. and Stefani, J. A. (1994), Supervisory runto-run control of polysilicon gate etch using in situ ellipsometry, IEEE Transactions on Semiconductor Manufacturing, 7(2), 193-201.

Carlson, A. and DiBiase, D. (2002), Effect of metrology time delay on overlay APC, Proceedings of SPIE, 4692, 1-16.

Chamness, K., Cherry, G., Good, R., and Qin, S. (2001), A comparison of $\mathrm{R} 2 \mathrm{R}$ control algorithms for the CMP with measurement delay, AEC/APC XIII Symposium, 1-4.

Chen, A. and Guo, R. (2001), Age-based double EWMA controller and its application to CMP processes, IEEE Transactions on Semiconductor Manufacturing, 14(1), 11-19.
Daniel, W. W. (1990), Applied Nonparametric Statistics, Boston.

del Castillo, E. and Hurwitz, A. M. (1997), Run-to-run process control: Literature review and extensions, Journal of Quality Technology, 29(2), 184-196.

del Castillo, E. and Yeh, J.-Y. (1998), An adaptive runto-run optimizing controller for linear and nonlinear semiconductor processes, IEEE Transactions on Semiconductor Manufacturing, 11(2), 285-295.

del Castillo, E. (1999), Long run and transient analysis of a double EWMA feedback controller, IIE Transactions, 31(12), 1157-1169.

del Castillo, E. (2001), Some properties of EWMA feedback quality adjustment schemes for drifting disturbances, Journal of Quality Technology, 33(2), 153-166.

Fan, S.-K. S., Jiang B. C., Jen, C.-H., and Wang, C.-C. (2002), SISO run-to-run feedback controller using triple EWMA smoothing for semiconductor manufacturing processes, International Journal of Production Research, 40, 3093-3120.

Good, R. P. and Qin, S. J. (2006), On the Stability of MIMO EWMA Run-to-Run Controllers with Metrology Delay, IEEE Transactions on Semiconductor Manufacturing, 19(1), 78-86.

Ingolfsson, A. and Sachs, E. (1993), Stability and sensitivity of an EWMA controller, Journal of Quality Technology, 25(4), 271-287.

Lo, L.-C. (2007), Adaptive Adjustment Scheme of Time-Varying Metrology Delay using RLS-type Controllers, master thesis, Department of Industrial Engineering and Management, Yuan Ze University, Taoyuan County, Taiwan 320.

Moyne, J., del Castillo, E., and Hurwitz. A. M. (2001), Run-to-Run Control in Semiconductor Manufacturing, Boca Raton, FL: CRC Press.

Siegel, S. and Castellan, N. J. (1988), Nonparametric systems for the Behavioral, Sciences, McGRAWHILL.

Wang, J., Qin, S. J., Bode, C. A., and Purdy, M. A. (2003), Recursive Least Squares Estimation and Its Application to Shallow Trench Isolation, Proceedings of SPIE-International Society for Optical Engineering, 5044, 109-120.

Wang, J., He, Q. P., Qin, S. J., Bode, C. A., and Purdy, M. A. (2005), Recursive Least Squares Estimation for Run-to-Run Control with Metrology Delay and Its Application to STI Etch Process, IEEE Transactions on Semiconductor Manufacturing, 18(2), 309318.

Wu, M. F., Lin, C. H., Wong, S. H., Jang, S. S., and Tseng, S. T. (2006), Performance Analysis of EWMA Controller Subject to Metrology Delay, AEC/APC Symposium Asia 2006, Taiwan. 


\section{APPENDIX I}

\section{Proof of Lemma 1}

Based on the process model and control action in Table 1 , the expected value of $y_{t}$ produced by using the DEWMA controller is

$$
\begin{aligned}
y_{t} & =\alpha+\beta u_{t-1}+\delta t+\varepsilon_{t} \\
& =\alpha+\beta\left(\frac{\tau-a_{t-t^{\prime}-1}-\left(1+t_{p}\right) D_{t-t^{\prime}-1}}{b}\right)+\delta t+\varepsilon_{t} \\
& =\alpha+\xi \tau-\xi a_{t-t^{\prime}-1}-\xi\left(1+t_{p}\right) D_{t-t^{\prime}-1}+\delta t+\varepsilon_{t} .
\end{aligned}
$$

As $t \rightarrow \infty$ and $\xi \cong 1$, the expected value of $y_{t}$ is

$$
\begin{aligned}
E\left(y_{t}\right) & =E\left(\alpha+\tau-a_{t-t^{\prime}-1}-D_{t-t^{\prime}-1}-t_{p} D_{t-t^{\prime}-1}+\delta t\right) \\
& =E\left(\tau-t_{p} D_{t-t^{\prime}-1}+\alpha+\delta t-a_{t-t^{\prime}-1}-D_{t-t^{\prime}-1}\right) \\
& =\tau-t_{p} \delta+t^{\prime} \delta \\
& =\tau+\delta\left(t^{\prime}-t_{p}\right)
\end{aligned}
$$

since $E\left(D_{t-1}\right) \rightarrow E\left(D_{t}\right), D_{t-1}$ is the estimate for $\delta$ and $a_{t-t^{\prime}-1}+D_{t-t^{\prime}-1}$ is the estimate for $\alpha+\delta\left(t-t^{\prime}\right)$. Therefore, six cases (B1-6) are followed.

\section{APPENDIX II}

\section{Proof of Lemma 2}

Based on the process model and control action in Table 1, the expected value of $y_{t}$ produced by using the RLS-LT controller is

$$
\begin{aligned}
& y_{t}=\alpha+\beta u_{t-1}+\delta t+\varepsilon_{t} \\
& =\alpha+\beta\left(\frac{\tau-\hat{\boldsymbol{\theta}}_{t_{t-t^{-1}}}-\hat{\boldsymbol{\theta}}_{t_{t-t_{-1}}}\left(1+\left(t-1-t^{\prime}\right)+t_{p}\right)}{b}\right)+\delta t+\varepsilon_{t} \\
& =\alpha+\xi \tau-\xi \hat{\boldsymbol{\theta}}_{1_{t-t^{-1}-1}}-\xi \hat{\boldsymbol{\theta}}_{2_{t-t^{\prime-1}}}\left(t-t^{\prime}+t_{p}\right)+\delta t+\varepsilon_{t} .
\end{aligned}
$$

As $t \rightarrow \infty$ and $\xi \cong 1$, the expected value of $y_{t}$ is

$$
\begin{aligned}
E\left(y_{t}\right) & =E\left(\alpha+\tau-\hat{\boldsymbol{\theta}}_{1_{t-t^{\prime}-1}}-\hat{\boldsymbol{\theta}}_{2_{t-t^{\prime}-1}}\left(t-t^{\prime}+t_{p}\right)+\delta t\right) \\
& =\tau+\delta\left(t^{\prime}-t_{p}\right)
\end{aligned}
$$

since $\hat{\theta}_{t_{t-t^{\prime}-1}} \hat{\boldsymbol{\theta}}_{\mathrm{t}_{t t^{\prime}-1}}$ is the estimate for $\alpha, \hat{\theta}_{2_{t t^{\prime}-1}}$ is the estimate for $\delta$, and $\hat{\boldsymbol{\theta}}_{\mathrm{1}_{t-t_{-1}}}+\hat{\boldsymbol{\theta}}_{t_{t-t^{-1}}}\left(t-t^{\prime}\right)$ is the estimate for $\alpha+\delta\left(t-t^{\prime}\right)$. Hence, six cases in (C1-6) are followed. 